M. Matsuda

\title{
Peripheral nerve involvement in primary systemic AL amyloidosis: a clinical and electrophysiological study
}

Masayuki Matsuda, MD, PhD*, Takahisa Gono, MD, PhD, Hiroshi Morita, MD, PhD,

Nagaaki Katoh, MD, PhD, Minori Kodaira, MD, Shu-ichi Ikeda, MD, PhD

Department of Medicine (Neurology and Rheumatology), Shinshu University School of Medicine, Matsumoto, Japan

Word-count for the paper: 3941 words

Rnning title: Polyneuropathy in AL amyloidosis

Correspondence: Masayuki Matsuda, MD, Department of Medicine (Neurology and Rheumatology), Shinshu University School of Medicine, 3-1-1 Asahi, Matsumoto 390-8621, Japan

Tel: $+81-263-37-2672$

Fax: $+81-263-37-3427$

E-mail: matsuma@shinshu-u.ac.jp

Keywords: AL amyloidosis, polyneuropathy, autonomic nerve dysfunction, carpal tunnel syndrome 
M. Matsuda

\begin{abstract}
Background: Involvement of visceral organs usually dominates the clinical picture of primary systemic AL amyloidosis, but some patients suffer from serious peripheral neuropathy. The aim of this study is to clinically and electrophysiologically investigate peripheral nerve involvement in AL amyloidosis patients.
\end{abstract}

Patients and methods: We reviewed clinical manifestations, electrophysiological findings including nerve conduction velocities, and treatments in 43 consecutive patients. Twenty age-matched healthy subjects were employed as controls.

Results: Fifteen patients (34.9\%) showed apparent neuropathic symptoms, which consisted of polyneuropathy in 11 (25.6\%), bilateral carpal tunnel syndrome in 4 (9.3\%), and autonomic dysfunction in 8 (18.6\%). Polyneuropathy in this disease was characterized by symmetrical and sensory-dominant impairment, early involvement of the lower limbs, loss of all sensations, rarity of motor weakness, and painful paresthesia in the legs predominant at an early stage. Autonomic dysfunction including orthostatic hypotension was frequently associated with polyneuropathy at an advanced stage. On electrophysiological studies MCV and CMAPs of both median and tibial nerves were significantly decreased in the patients with polyneuropathy but also in those without any signs of neuropathy. Only four of 15 patients with neuropathy were able to receive intensive but promising chemotherapy with a large dose of melphalan for plasma cell dyscrasia.

Conclusions: Peripheral nerves in primary systemic AL amyloidosis patients seem to be involved more extensively than clinical manifestations might suggest. The clinical picture of polyneuropathy in this disease closely resembles that in transthyretin-type familial amyloid polyneuropathy patients with a late age at onset, particularly those originating from sporadic kindreds. 
M. Matsuda

\section{INTRODUCTION}

Primary systemic amyloidosis is a chronic hematological disorder caused by plasma cell dyscrasia or B-cell lymphoproliferative disorders. Amyloid fibrils derived from immunoglobulin light chains (AL) gradually accumulate in multiple vital organs, including the heart, kidneys, liver, and gastrointestinal tract. Because of progressive dysfunction of these organs the prognosis of primary systemic AL amyloidosis is usually bad if left untreated [1]. High-dose melphalan with autologous peripheral blood stem cell transplantation (HDM/SCT) has been recently reported to show good therapeutic effects on both hematological response and organ function of the patients with this disease [2-4], leading to an increase in their overall survival rate, although it remains controversial whether this treatment is truly superior to other intensive chemotherapies or not [5]. Peripheral nerves are also affected in patients with primary systemic AL amyloidosis and some of them manifest sensory-dominant polyneuropathy and/or autonomic dysfunction [6, 7], but the detailed pathophysiological conditions of peripheral nerve impairment in this disease remain unclear.

In this study we retrospectively studied peripheral nerve involvement in primary systemic AL amyloidosis patients with regard to clinical findings, neurophysiological parameters including nerve conduction velocities and coefficients of variation of R-R intervals $\left(\mathrm{CV}_{\mathrm{R}-\mathrm{R}}\right)$, and their selected chemotherapies.

\section{PATIENTS AND METHODS}

\section{$\underline{\text { Subjects }}$}

We retrospectively studied 43 primary systemic AL amyloidosis patients who had been consecutively admitted to our hospital between April 2002 and June 2008 (28 men and 15 women; age range, 43 to 71 years; mean, $57.0 \pm 7.3$ years). None of the patients had received any medical treatments for plasma cell dyscrasia prior to being referred to us. The diagnosis of this type of amyloidosis was based on the criteria proposed by 
M. Matsuda

Gertz et al [8]. To detect amyloid deposition, conventional alkaline Congo red staining was performed in several biopsied tissues such as the kidney and the gastroduodenal or rectal mucosa, and in at least one of these specimens immunohistochemical staining was

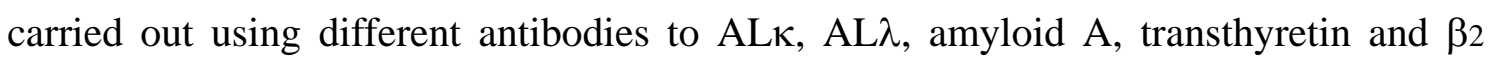
microglobulin in order to confirm it as AL type [9].

We obtained information on clinical findings including neurological examinations, results of electrophysiological studies, and treatments from the medical records of the patients. The presence of visceral organ involvement was assessed in each patient based on physical examinations and laboratory data, such as daily protein excretion in urine, hepatobiliary enzymes in serum and electro- and echocardiogram. Intensive chemotherapy for plasma cell dyscrasia, HDM/SCT, was performed according to our eligibility criteria [4]. To compare the results of electrophysiological studies, 20 age-matched healthy subjects (13 men and 7 women; age range, 45 to 68 years; mean, $56.0 \pm 5.9$ years) were employed as controls. The Committee for Medical Ethics of Shinshu University School of Medicine approved this study.

\section{Electrophysiological studies}

Nerve conduction studies were carried out in median and tibial nerves. When clinical symptoms were not completely symmetrical, we selected the predominantly affected side. Terminal latency, negative amplitude of the compound muscle action potential (CMAP) and maximal motor conduction velocity (MCV) were measured and calculated in both median and tibial nerves. An active surface electrode was placed on the muscle belly and a reference electrode on the distal tendon to record CMAP of the abductor pollicis brevis muscle (APB) and abductor hallucis muscle (AH). The median nerve was stimulated at the wrist $(7.0 \mathrm{~cm}$ proximal to the active recording electrode over the APB) and elbow. Tibial nerve was stimulated at the ankle (internal malleolus, $10.0 \mathrm{~cm}$ proximal to the active recording electrode over the $\mathrm{AH}$ ) and knee (popliteal fossa). 
M. Matsuda

To measure the maximal sensory nerve conduction velocity (SCV) of both the median and tibial nerves using common antidromic methods, pairs of surface recording electrodes were placed on the second finger and the third toe. Median and tibial nerves were stimulated in the same manner as for the motor conduction study. Peak to peak amplitude of sensory nerve action potential (SNAP) and SCV of both distal (finger-wrist or toe-ankle) and proximal (wrist-elbow or ankle-knee) parts were measured. When we could not evoke SNAP with antidromic methods using surface recording electrodes, we employed the near-nerve method. The stimulating surface ring electrodes were placed around the second finger and the third toe, and pairs of needle recording electrodes were insulated at the wrist, elbow, internal malleolus, and popliteal fossa. $\mathrm{CV}_{\mathrm{R}-\mathrm{R}}$ on electrocardiogram was measured for evaluation of cardiac autonomic nerve function.

$\underline{\text { Statistics }}$

To determine whether statistically significant differences existed, we used Student's t-test and Mann-Whitney's U test. A simple regression test was used for detection of a significant relationship between $\mathrm{CV}_{\mathrm{R}-\mathrm{R}}$ and parameters of electrophysiological studies. The results represent the mean \pm standard deviation where applicable, and a p-level less than 0.05 was considered to be statistically significant. Commercially available statistics software was used for data analysis (StatView for Macintosh, Abacus Concepts, Berkeley, CA, USA).

\section{RESULTS}

\section{$\underline{\text { Clinical and laboratory features }}$}

Fifteen patients (34.9\%) of the 43 examined showed clinically apparent neuropathic symptoms due to peripheral nerve involvement. Their clinical profiles are shown in Table 1. Nine were men and 6 were women. Age at diagnosis of peripheral nerve involvement and disease duration were 57.9 \pm 8.7 years and $14.7 \pm 7.2$ months, 
M. Matsuda

respectively. Four patients showed neuropathic symptoms as a predominant clinical manifestation, while the remaining 11 presented with a mixture of visceral and neuropathic symptoms. Disease duration at diagnosis was $17.5 \pm 6.4$ months in the former group and $13.7 \pm 7.5$ months in the latter, although there was no significant difference between them. In the latter group clinical symptoms due to nephrotic syndrome and congestive heart failure frequently overshadowed the neuropathy. Ten had weight loss, general fatigue and light-headedness; 5 had edema, and the same number had dyspnea on exertion. Four complained of other symptoms, such as nausea, back pain, and hematuria. Physical examination revealed mild hepatomegaly in 2 patients, purpura in 2, macroglossia in 6, and pretibial edema in 7.

Laboratory data demonstrated abnormalities in all these 15 patients. Proteinuria was present in 12 patients, 5 of whom showed nephrotic syndrome. Three had serum creatinine levels greater than $1.5 \mathrm{mg} / \mathrm{dl}$, and eight showed serum hemoglobin levels less than $12 \mathrm{~g} / \mathrm{dl}$. None of the patients showed increases in aspartate aminotransferase, alanine aminotransferase or alkaline phosphatase. Bone marrow aspirates were obtained from all the patients. The ratio of plasma cells in bone marrow was $5.0 \pm 3.4 \%$. Serum and urine immunofixation revealed positive results in 9 and 14 patients, respectively. M-protein in serum was $\operatorname{IgG} \lambda$ in 3 patients, $\operatorname{IgA\kappa }$ in 1 , $\operatorname{IgA} \lambda$ in $1, \operatorname{IgD} \lambda$ in 1 , and Bence Jones protein $\lambda$ in 3 . One patient (case 4) showed no M-protein in either serum or urine, but the analysis of free light chains in serum and flow cytometry revealed clonal expansion of plasma cells [10]. To make a definite diagnosis of AL amyloidosis on immunohistochemical staining, renal biopsy was performed in 7 patients because of proteinuria. Other biopsy sites were gastroduodenum in 6 patients, rectum in 2, and gingival mucosa in 1 . Sural nerve biopsy was performed in only one patient with polyneuropathy, and showed massive deposits of amyloid with a reduced number of myelinated nerve fibers. All amyloid deposits on these biopsied tissues were specifically

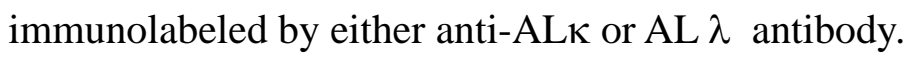


M. Matsuda

$\underline{\text { Neurological features }}$

Sensory disturbance was most prominent in all 15 patients with neuropathic symptoms except for one with autonomic dysfunction alone. No patients showed cranial nerve involvement. Eleven patients (7 men and 4 women) were clinically diagnosed as having polyneuropathy, 4 of them suffering mainly from this disorder. They showed more marked involvement of the lower limbs than the upper. Painful paresthesia and loss of both cutaneous (pain and thermal sense) and discriminative (vibration and position sense) sensations were seen in all of these 11 patients. Motor weakness localized to the lower legs was present only in 3 patients at an advanced stage. Two of the 11 patients showed polyneuropathy as the initial symptom of AL amyloidosis. Three patients had numbness only in the hands, and one with polyneuropathy developed this symptom in his hands before involvement of his feet. These 4 patients ( 2 men and 2 women) were diagnosed as having bilateral CTS based on the distribution of numbness and positive Tinel's sign on the wrist.

Eight patients (5 men and 3 women; 18.6\%), including 7 with polyneuropathy, complained of autonomic symptoms: all of them had orthostatic hypotension, which frequently impaired their activities of daily living. Other manifestations were decreased sweating in 3 patients, urinary incontinence in 2, and alternating constipation and diarrhea in 1. Four of 5 male patients showed impotence. No patient suffered from periodic severe nausea or vomiting. In all 7 patients with both polyneuropathy and autonomic dysfunction the former symptom always preceded the latter. Only one patient had mild orthostatic hypotension alone.

\section{Electrophysiological studies}

Results of electrophysiological studies in primary systemic AL amyloidosis patients and controls are summarized in Table 2. In the median nerve, whole patients and those with polyneuropathy showed a significant elongation of terminal latency and significant decreases in MCV, CMAP, distal SCV and SNAP compared with controls. 
M. Matsuda

MCV, CMAP, proximal and distal SCV, and SNAP were significantly decreased and terminal latency was significantly longer also in the patients without clinically apparent neuropathic symptoms compared with controls. The patients with polyneuropathy tended to show lower levels of MCV, CMAP, SCV and SNAP than those without neuropathic symptoms, but there were no significant statistical differences between the two groups.

In the tibial nerve, whole patients and those with polyneuropathy showed significant decreases in MCV, CMAP and SCV compared with controls. Terminal latency was significantly longer in whole patients than in controls. The patients with polyneuropathy also showed a longer mean value of terminal latency compared with controls, although no significant differences between the two groups were present. The patients without neuropathic symptoms revealed a significant elongation of terminal latency and significant decreases in MCV, CMAP and proximal SCV compared with controls. The patients with polyneuropathy tended to show lower levels of MCV, CMAP and SCV than those without neuropathic symptoms, but there were no significant statistical differences between the two groups.

In the median nerve of the patients with bilateral CTS, MCV, CMAP, proximal and distal SCV, and SNAP showed lower levels and the mean value of terminal latency was longer compared with controls. No apparent change was present in the tibial nerve. These findings were compatible with the clinical diagnosis of CTS.

$\mathrm{CV}_{\mathrm{R}-\mathrm{R}}$ was significantly lower in whole patients, those without neuropathic symptoms and those with polyneuropathy than in controls. The patients with polyneuropathy showed significantly lower $\mathrm{CV}_{\mathrm{R}-\mathrm{R}}$ than those without neuropathic symptoms. There was significantly positive correlations between $\mathrm{CV}_{\mathrm{R}-\mathrm{R}}$ and $\mathrm{MCV}$ $(\mathrm{p}<0.05)$ or CMAP $(\mathrm{p}<0.01)$ in the tibial nerve of the patients with peripheral nerve involvement.

$\underline{\text { Treatment }}$ 
M. Matsuda

HDM/SCT was performed in 20 of the whole 43 patients (46.5\%): four of the 15 patients with peripheral neuropathy (26.7\%) received HDM/SCT, while 16 of the 28 patients without it (57.1\%) were treated with this intensive chemotherapy. Poor performance status due to disease progression was the most common reason why the remaining patients could not undergo HDM/SCT, and advanced age was the second. With regard to the 4 patients showing polyneuropathy as a main disorder, 2 were treated with HDM/SCT, the details of which have been reported elsewhere [11]. However, the other 2 did not receive this treatment since both were in an almost bed-ridden state due to severe orthostatic hypotension. Eleven patients with peripheral neuropathy who were ineligible for HDM/SCT received other chemotherapies: VAD (vincristine, doxorubicin and dexamethasone) alone [12] in 5, M-dex (oral melphalan and large doses of dexamethasone) [13] in 4 and MP (oral melphalan and prednisolone) in 2. Vincristine has neurotoxicity as an adverse effect, but none of the patients treated with VAD showed apparent worsening of neuropathic symptoms.

\section{DISCUSSION}

Amyloid neuropathy is usually divided into two forms [14]: one is familial amyloid polyneuropathy (FAP), in which the precursor protein is transthyretin (TTR), and the other is peripheral nerve involvement associated with primary systemic AL amyloidosis. FAP with amyloid derived from TTR (ATTR) was once considered a disease peculiar to endemic areas, but FAP kindreds are now known to exist in many nations worldwide and ATTR-type FAP is recognized not to be a rare disease [15]. The clinical and pathophysiological similarity or difference among ATTR-type FAP and AL amyloidosis-related peripheral neuropathy is still incompletely understood. To address this clinical issue, amyloid typing is of basic importance. In this study we analyzed clinical symptoms and peripheral nerve parameters in AL amyloidosis patients, all of

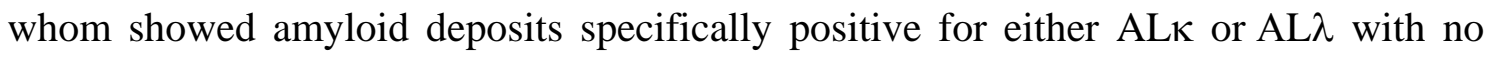


immunoreactivity for ATTR irrespective of the presence of M-protein.

Clinical symptoms due to AL amyloid-induced peripheral nerve impairment in the present study consisted of polyneuropathy, bilateral CTS and autonomic dysfunction. The mean age at diagnosis of peripheral nerve involvement was 57.9 years, and two thirds of the patients were male. Approximately one third of the whole patients examined in this study showed one or two of these neuropathic symptoms. Polyneuropathy was seen in approximately one fourth of them and autonomic dysfunction was highly concurrent in these patients. This frequency was compatible with that of a previous report from another institute $[1,6]$. A notable finding was the considerable similarity in the clinical picture of peripheral neuropathy between primary systemic AL amylodiosis and ATTR-type FAP. In the present study 11 patients were affected by polyneuropathy, which started in the legs with painful paresthesia and progressed in an ascending fashion, showing symmetrical and sensory-dominant impairment: all sensations were equally affected, and motor weakness and autonomic dysfunction appeared at an advanced stage. Within the autonomic dysfunction, orthostatic hypotension was the most common symptom, while severe digestive symptoms including periodic nausea and vomiting were not seen. The clinical phenotypes of ATTR-type FAP are reported to vary in different kindreds or individuals with diverse mutations of TTR genes $[16,17]$ : the presence of dissociated sensory loss (an initial and selective involvement of pain and thermal sensations) and autonomic failure at an early stage of the disease has been emphasized to be a cardinal feature in ATTR-type FAP patients from endemic foci $[15,18]$. However, this is not seen in FAP patients from sporadic kindreds, even in those with ATTR Val30Met (methionine is substituted for valine at position 30 of TTR) showing a late age of onset and male predominance $[15,18]$. Additionally, CTS is frequently associated with FAP patients irrespective of various TTR gene mutations $[16,19]$. Thus, the overall clinical manifestations of peripheral neuropathy ascribed to primary systemic AL amyloidosis 
M. Matsuda

including the age of onset and sex ratio are very similar to those in ATTR-type FAP patients originating from non-endemic areas $[15,18]$.

Another notable finding in the present study was that the peripheral nervous system seemed to be more extensively affected in the patients with this disease than was manifested clinically. In our series of patients neuropathic symptoms were seen at a frequency of approximately 35\%: 11 patients with polyneuropathy commonly showed significant reduction of MCV, CMAP, SCV and SNAP in both median and tibial nerves, and 4 patients with clinically definite CTS showed decreases in both distal and proximal SCV of the median nerve, indicating diffuse deposition of AL amyloid on the peripheral nervous system in these patients [20]. Additionally in the patients without clinical manifestations of peripheral neuropathy, MCV and CMAP were obviously decreased in both median and tibial nerves compared with those of controls. SCV and SNAP in the median nerve were also significantly lower in these patients than in controls. $\mathrm{CV}_{\mathrm{R}-\mathrm{R}}$, which reflects cardiac parasympathetic nerve function [21], showed a significant decrease both in the patients with apparent peripheral neuropathy and in those without, compared with controls: in approximately $60 \%$ of the whole patients abnormal values of $\mathrm{CV}_{\mathrm{R}-\mathrm{R}}$ of less than $2.0 \%$ were observed irrespective of the presence of autonomic symptoms. All these results suggest that subclinical involvement of peripheral somatic and autonomic nerves is more frequent in primary systemic AL amyloidosis patients than previously reported $[1,6]$ and that electrophysiological examinations are useful for detecting these latent peripheral nerve lesions.

Finally, we emphasize that primary systemic AL amyloidosis is now a treatable disease [22] and that AL amyloidosis patients with peripheral neuropathy alone are the most suitable for intensive chemotherapy with HDM/SCT, targeting an underlying disorder such as plasma cell dyscrasia [23, 24]. A few patients who received this therapy have already shown recovery of peripheral neuropathy [11, 23, 24], providing histopathological evidence of AL amyloid regression [11]. In the present study, however, 
M. Matsuda

HDM/SCT was performed in only $26.7 \%$ of the patients with peripheral neuropathy. This figure is quite low in comparison with $57.1 \%$ in the patients without neuropathic symptoms. The most common reason why HDM/SCT was not performed in the patients with peripheral neuropathy was disease progression, and particularly in those with polyneuropathy poor performance status due to severe orthostatic hypotension made this treatment inapplicable. Eligibility for HDM/SCT in patients with primary systemic AL amyloidosis usually depends on visceral organ function, especially on preserved cardiac function [8], but the present study has indicated that the severity of peripheral neuropathy including autonomic dysfunction is an important determinant in whether or not patients should undergo this risk-adapted chemotherapy.

Acknowledgments: This study was supported by grants from Neuroimmunological Disease Division and Amyloid Research Committee, the Ministry of Public Health, Labor and Welfare, Japan.

None of the authors declare any conflict of interest. 
M. Matsuda

\section{REFERENCES}

[1] Kyle RA, Gertz MA. Primary systemic amyloidosis. Clinical and laboratory features in 474 Cases. Semin Hematol 1995; 32: 45-59.

[2] Comenzo RL, Vosburgh E, Simms RW, et al. Dose-intensive melphalan with blood stem cell support for the treatment of AL amyloidosis: one-year follow-up in five patients. Blood 1996; 88: 2801-6.

[3] Skinner M, Sanchorawala V, Seldin DC,.et al. High-dose melphalan and autologous stem-cell transplantation in patients with AL amyloidosis: an 8-year study. Ann Intern Med 2004; 140: 85-93.

[4] Gono T, Matsuda M, Shimojima Y, et al. VAD with or without subsequent high-dose melphalan followed by autologous stem cell support in AL amyloidosis: Japanese experience and criteria for patient selection. Amyloid: J Protein Folding Disord 2004; 11: 245-56.

[5] Jaccard A, Moreau P, Leblond V, et al. High-dose melphalan versus melphalan plus dexamethasone for AL amyloidosis. N Engl J Med 2007; 357: 1083-93.

[6] Kelly JJ, Kyle RA, O’Brien PC, Dyck PJ. The natural history of peripheral neuropathy in primary systemic amyloidosis. Ann Neurol 1979; 6: 1-7.

[7] Rajkumar SV, Gertz MA, Kyle RA. Prognosis of patients with primary systemic amyloidosis who present with dominant neuropathy. Am J Med 1998; 104: 232-237

[8] Gertz MA, Comenzo R, Falk RH, et al. Definition of organ involvement and treatment response in immunoglobulin light chain amyloidosis (AL): a consensus opinion from the $10^{\text {th }}$ international symposium on amyloid and amyloidosis. Am J Hematol 2005; 79: 319-28.

[9] Hoshii Y, Kiyama M, Cui D, Kawano H, Ishihara T. Immunohistochemical study of immunoglobulin light chain amyloidosis with antibodies to the immunoglobulin light chain variable region. Pathol Int 2006; 56: 324-30. 
M. Matsuda

[10] Yoshida T, Matsuda M, Katoh N, et al. Long-term follow-up of plasma cells in bone marrow and serum free light chains in primary systemic AL amyloidosis. Intern Med 2008; 47: 1783-90.

[11] Katoh N, Matsuda M, Yoshida T, et al. Primary AL amyloid polyneuropathy successfully treated with high-dose melphalan followed by autologous stem cell transplantation. Muscle Nerve 2010; 41: 138-43.

[12] Matsuda M, Gono T, Katoh N, et al. Nephrotic syndrome due to primary systemic AL amyloidosis, successfully treated with VAD (vincristine, doxorubicin and dexamethasone) alone. Intern Med 2008; 47: 543-49.

[13] Palladini G, Perfetti V, Obici L, et al. Association of melphalan and high-dose dexamethasone is effective and well tolerated in patients with AL (primary) amyloidosis who are ineligible for stem cell transplantation. Blood 2004; 103 : 2936-8.

[14] Kyle RA, Dyck PJ. Amyloidosis and neuropathy. In: Dyck PJ, Thomas PK, Griffin JW, Low PA, Poduslo JF, editors. Peripheral neuropathy, $3^{\text {rd }}$ ed. Philadelphia: W.B. Saunders; 1993. p 1294-309.

[15] Ikeda S, Nakazato M, Ando Y, Sobue G. Familial transthyretin-type amyloid polyneuropoathy in Japan. Clinical and genetic heterogeneity. Neurology 2002; 58: $1001-7$.

[16] Connors LH, Lim A, Prokaeva T, Roskens VA, Costello CE. Tabulation of human transthyretin (TTR) variants, 2003. Amyloid: J Protein Folding Disord 2003; 10: 160-84.

[17] Benson MD, Kincaid JC. The molecular biology and clinical features of amyloid neuropathy. Muscle Nerve 2007; 36: 411-23.

[18] Koike H, Misu K, Ikeda S, et al. Type 1 (transthyretin Met30) familial amyloid polyneuropathy in Japan. Early- vs late-onset form. Arch Neurol 2002; 59: 1771-6. 
M. Matsuda

[19] Koike H, Morozumi S, Kawagashira Y, et al. The significance of carpal tunnel syndrome in transthyretin Val30Met familial amyloid polyneuropathy. Amyloid: J Protein Folding Disord 2009; 16: 142-8.

[20] Tojo K, Tsuchiya-Suzuki A, et al. Upper limb neuropathy such as carpal tunnel syndrome as an initial manifestation of ATTR Val30Met familial amyloid polyneuropathy. Amyloid: J Protein Folding Disord 2010; 17: 32-5.

[21] Ando Y, Araki S, Shimoda O, Kano T. Role of autonomic nerve functions in patients with familial amyloidotic polyneuropathy as analyzed by laser doppler flowmetry, capsule hydrograph, and cardiographic R-R interval. Muscle Nerve 1991; 15: 507-12.

[22] Sanchorawala V, Seldin DC. An overview of high-dose melphalan and stem cell transplantation in the treatment of AL amyloidosis. Amyloid: J Protein Folding Disord 2007; 14: 261-9.

[23] Comenzo RL, Vosburgh E, Simms RW, et al. Dose-intensive melphalan with blood stem cell support for the treatment of AL amyloidosis: one-year follow-up in five patients. Blood 1996; 88: 2801-6.

[24] Skinner M, Sanchorawala V, Seldin DC, et al. High-dose melphalan and autologous stem-cell transplantation in patients with AL amyloidosis: an 8-year study. Ann Intern Med 2004; 140: 85-93. 
Table 1. Clinical profiles of the patients with peripheral nerve involvement

\begin{tabular}{|c|c|c|c|c|c|c|c|c|c|c|c|c|c|}
\hline \multirow[b]{2}{*}{ Case } & \multirow[b]{2}{*}{ Age/Sex } & \multirow{2}{*}{$\begin{array}{l}\text { Disease } \\
\text { duration } \\
\text { (months) }\end{array}$} & \multicolumn{2}{|c|}{ M-protein } & \multicolumn{3}{|c|}{ Immunohistochemistry } & \multirow{2}{*}{$\begin{array}{l}\text { Symptoms due } \\
\text { to visceral } \\
\text { organ } \\
\text { involvement }\end{array}$} & \multicolumn{3}{|c|}{ Neurological symptoms } & \multirow[b]{2}{*}{$\begin{array}{c}\text { Performance } \\
\text { status* }\end{array}$} & \multirow[b]{2}{*}{ Treatment } \\
\hline & & & Serum & Urine & Aк & $\mathrm{A} \lambda$ & ATTR & & $\begin{array}{c}\text { Carpal } \\
\text { tunnel } \\
\text { syndrome }\end{array}$ & Polyneuropathy & $\begin{array}{l}\text { Autonomic } \\
\text { symptoms }\end{array}$ & & \\
\hline 1 & $43 / \mathrm{F}$ & 24 & IgA $\lambda$ & BJP $\lambda$ & - & + & - & - & - & + & + & 2 & HDM/SCT \\
\hline 2 & $44 / F$ & 23 & $\operatorname{IgG} \lambda$ & BJP $\lambda$ & - & + & - & CHF & + & + & - & 3 & VAD \\
\hline 3 & $48 / \mathrm{M}$ & 22 & - & ВJPк & + & - & - & - & - & + & + & 2 & HDM/SCT \\
\hline 4 & $49 / \mathrm{F}$ & 5 & - & - & + & - & - & NS, CHF & - & + & + & 3 & M-dex \\
\hline 5 & $54 / \mathrm{M}$ & 15 & - & BJP $\lambda$ & - & + & - & NS & - & + & + & 4 & VAD \\
\hline 6 & $55 / F$ & 16 & - & BJP $\lambda$ & - & + & - & NS & - & + & - & 2 & HDM/SCT \\
\hline 7 & $58 / \mathrm{M}$ & 12 & - & BJP $\lambda$ & - & + & - & - & - & + & + & 4 & M-dex \\
\hline 8 & $61 / \mathrm{F}$ & 5 & IgAK & ВЈPк & + & - & - & NS & - & - & + & 3 & VAD \\
\hline 9 & $62 / \mathrm{F}$ & 19 & $\operatorname{IgG} \lambda$ & BJP $\lambda$ & - & + & - & CHF & + & - & - & 4 & MP \\
\hline 10 & $62 / \mathrm{M}$ & 3 & $\operatorname{IgD} \lambda$ & BJP $\lambda$ & - & + & - & CHF & - & + & - & 3 & M-dex \\
\hline 11 & $64 / \mathrm{M}$ & 17 & BJP $\lambda$ & BJP $\lambda$ & - & + & - & CHF & + & - & - & 2 & HDM/SCT \\
\hline 12 & $66 / M$ & 6 & BJP $\lambda$ & BJP $\lambda$ & - & + & - & Hematuria & + & - & - & 2 & VAD \\
\hline 13 & $66 / \mathrm{M}$ & 20 & BJP $\lambda$ & BJP $\lambda$ & - & + & - & CHF & - & + & - & 3 & M-dex \\
\hline 14 & $68 / \mathrm{M}$ & 22 & $\operatorname{IgG} \lambda$ & BJP $\lambda$ & - & + & - & NS & - & + & + & 2 & VAD \\
\hline 15 & 69/M & 12 & - & BJP $\lambda$ & - & + & - & - & - & + & + & 3 & MP \\
\hline
\end{tabular}

*Southwestern Oncology Group (SWOG) performance status [2]. ATTR: amyloid derived from transthyretin,

BJP: Bence Jones protein, CHF: congestive heart failure, HDM/SCT: high-dose melphalan with stem cell transplantation,

M-dex: melphalan and dexamethasone, MP: melphalan and prednisolone, NS: nephrotic syndrome,

VAD: vincristine, doxorubicin and dexamethasone 
Table 2. Results of electrophysiological studies in patients with primary systemic AL amyloidosis

\begin{tabular}{|c|c|c|c|c|c|c|c|}
\hline & & & $\begin{array}{c}\text { Whole patients } \\
\qquad(\mathrm{n}=43)\end{array}$ & $\begin{array}{l}\text { Patients without clinically apparent } \\
\text { neuropathic symptoms } \\
(\mathrm{n}=28)\end{array}$ & $\begin{array}{c}\text { Patients with } \\
\text { polyneuropathy } \\
(\mathrm{n}=11)\end{array}$ & $\begin{array}{l}\text { Patients with CTS } \\
\qquad(\mathrm{n}=4)\end{array}$ & $\begin{array}{c}\text { Age-matched } \\
\text { controls } \\
(\mathrm{n}=20)\end{array}$ \\
\hline \multirow[t]{18}{*}{ Median nerve } & Terminal latency & Mean (ms) & 4.2 & 4.3 & 4.0 & 8.9 & 3.3 \\
\hline & & $\mathrm{SD}$ & 2.34 & 2.67 & 0.68 & 6.10 & 0.31 \\
\hline & & vs. controls & $<0.0001 *$ & $0.0001 *$ & 0.003* & & \\
\hline & $\overline{\mathrm{MCV}}$ & Mean $(\mathrm{m} / \mathrm{s})$ & 55.2 & 55.7 & 53.8 & 46.0 & 60.3 \\
\hline & & SD & 6.18 & 6.34 & 5.75 & 6.48 & 3.35 \\
\hline & & vs. controls & 0.0013 & 0.008 & 0.006 & & \\
\hline & $\overline{\text { CMAP }}$ & Mean (mV) & 6.5 & 6.8 & 5.2 & 2.1 & 9.5 \\
\hline & & SD & 3.40 & 3.67 & 1.88 & 1.43 & 2.59 \\
\hline & & vs. controls & 0.003 & 0.013 & $0.0002 *$ & & \\
\hline & $\mathrm{SCV}$ & Mean $(\mathrm{m} / \mathrm{s})$ & 49.0 & 49.1 & 49.0 & 27.0 & 56.3 \\
\hline & (finger-wrist) & SD & 10.35 & 11.42 & 6.47 & 8.6 & 5.36 \\
\hline & & vs. controls & 0.0003* & 0.014 & 0.0099 & & \\
\hline & $\mathrm{SCV}$ & Mean $(\mathrm{m} / \mathrm{s})$ & 62.2 & 62.2 & 62.0 & 53.0 & 68.1 \\
\hline & (wrist-elbow) & SD & 7.01 & 6.57 & 8.62 & 8.45 & 6.58 \\
\hline & & vs. controls & 0.008 & 0.011 & 0.074 & & \\
\hline & $\overline{\text { SNAP }}$ & Mean $(\mu \mathrm{V})$ & 6.1 & 6.5 & 4.7 & 1.0 & 15.3 \\
\hline & & SD & 4.09 & 4.43 & 2.58 & 2.05 & 0.55 \\
\hline & & vs. controls & $<0.0001$ & $<0.0001$ & $<0.0001$ & & \\
\hline \multirow[t]{15}{*}{ Tibial nerve } & Terminal latency & Mean (ms) & 4.7 & 4.6 & 5.1 & 4.4 & 4.1 \\
\hline & & SD & 1.13 & 0.83 & 1.70 & 0.88 & 0.58 \\
\hline & & vs. controls & 0.0005* & 0.0012* & 0.096 & & \\
\hline & $\overline{\mathrm{MCV}}$ & Mean $(\mathrm{m} / \mathrm{s})$ & 44.7 & 45.6 & 42.5 & 44.3 & 48.8 \\
\hline & & SD & 4.52 & 3.79 & 5.72 & 4.35 & 2.53 \\
\hline & & vs. controls & 0.0007 & 0.0046 & $0.0006^{*}$ & & \\
\hline & $\overline{\text { CMAP }}$ & Mean (mV) & 9.2 & 10.6 & 4.9 & 9.1 & 13.5 \\
\hline & & SD & 5.09 & 4.62 & 4.05 & 6.51 & 2.27 \\
\hline & & vs. controls & 0.0007 & 0.016 & $<0.0001$ & & \\
\hline & SCV & Mean $(\mathrm{m} / \mathrm{s})$ & 39.5 & 42.2 & 31.3 & 39.9 & 42.6 \\
\hline & (3rd toe-ankle) & SD & 11.37 & 6.87 & 17.67 & 5.55 & 3.68 \\
\hline & & vs. controls & $0.025 *$ & $0.13^{*}$ & 0.0051* & & \\
\hline & $\overline{S C V}$ & Mean $(\mathrm{m} / \mathrm{s})$ & 49.4 & 53.5 & 37.0 & 52.8 & 56.6 \\
\hline & (ankle-knee) & SD & 14.16 & 7.17 & 21.94 & 6.24 & 7.89 \\
\hline & & vs. controls & 0.0057* & 0.033* & 0.0007* & & \\
\hline \multirow[t]{3}{*}{$\overline{C V_{R}-R}$} & & Mean (\%) & 1.64 & 1.92 & 0.99 & 1.53 & 3.01 \\
\hline & & SD & 0.92 & 0.92 & 0.57 & 1.03 & 0.52 \\
\hline & & vs. controls & $<0.0001$ & $<0.0001$ & $<0.0001$ & & \\
\hline
\end{tabular}

*Statistical difference was evaluated by Mann-Whitney's U test because of non-normal distribution.

CMAP: compound muscle action potential, MCV: motor nerve conduction velocity, SCV: sensory nerve conduction velocity,

SD: standard deviation, CTS: carpal tunnel syndrome, NS: not significant 